seerechtliche Staatenpraxis der neun Anlieger der südostasiatischen Region dargestellt (Brunei, Burma, Kambodscha, Indonesien, Malaysia, Philippinen, Singapur, Thailand und Vietnam), ohne die wichtigsten Nachbarn wie Indien, China und Australien zu ignorieren.

Wie der Titel zeigt, stehen die Seegrenzen im Mittelpunkt. Sie werden nach zwei einführenden Kapiteln in richtiger logischer Reihenfolge unter den Gesichtspunkten der Basislinien, des Küstenmeeres, der Ausschließlichen Wirtschaftszone, der Inseln, der Archipele und der halbgeschlossenen Meere, mithin der entsprechenden Ordnungskriterien des neuen Seerechts behandelt.

Der Informationswert der Arbeit liegt darin, daß der Verfasser für jedes dieser Kapitel die gesamte Staatenpraxis der Region berichtet, dem geltenden Seerecht gegenüberstellt und bewertet. Die Kapitel über Basislinien und über Archipele ragen heraus, da sie die für diese Region typischen geographischen Faktoren wie große und kleinste Inseln, Halbinseln, verwinkelte Küsten usw. in ihren seerechtlichen Auswirkungen zeigen. Hierbei scheut der Verfasser nicht zurück vor kritischen Urteilen über Rechtsverstöße und exzessive Praxis beinahe aller Staaten der Region.

Das Bemühen um Vollständigkeit der jeweiligen nationalen und örtlichen Besonderheiten, der umstrittenen Inseln und der aus der Kolonialzeit überkommenen Tatbestände und Regelungslücken verdient hohe Anerkennung. Der Verfasser kritisiert regelmäßig die überzogenen Ansprüche einzelner Staaten. Das Buch ist damit - nicht zuletzt auch wegen der vielen Karten, Tabellen und abgedruckten Dokumente - eine Fundgrube für die seerechtliche Analyse der Staaten Südostasiens, die mit einer weit überdurchschnittlichen Zahl von Seegrenzproblemen leben müssen. Darüber hinaus ist zu hoffen, daß die Dokumentation von überzogenen Seerechtsansprüchen für Wissenschaft, Politik und Diplomatie ein Anreiz ist, dem gröbsten Wildwuchs energischer als bisher zu widersprechen, durch rechtswahrende Praxis gewohnheitsrechtliche Absonderlichkeiten zu verhindern und im übrigen friedliche Methoden der Beilegung von Grenzstreitigkeiten zu verfolgen.

Uwe Jenisch

\title{
Dieter Dörr
}

Die deutsche Handelsflotte und das Grundgesetz

München 1988, 289 S., DM 96,-

Von der Romantik alter Teeklipper ist in der vom harten Wettbewerb geprägten modemen Handelsschiffahrt kaum etwas geblieben. Zwar muß die Hanse noch regelmäßig als Vorbild für alle möglichen (angestrebten) Institutionen herhalten, doch lassen die aktuellen Probleme des Seetransports historische Anleihen zumeist nicht als sehr sinnvoll erscheinen. Bei Themen wie den Gefahrguttransporten oder dem umstrittenen Zweitregister stehen 
komplexe Sachverhalte zur Regelung an, die auf nationaler wie internationaler Ebene vielfältige Interessen berühren. Trotz der ökonomischen Bedeutung des Seetransports ist das betreffende Rechtsgebiet eine Angelegenheit für Spezialisten geblieben. Dies gilt auch für die bislang wenig beachtete Verfassungsnorm des Art. 27 GG, die bestimmt, daß alle deutschen Kauffahrteischiffe eine einheitliche Handelsflotte bilden. Es ist nun das Verdienst von Dieter Dörr, mit seiner 1987 abgeschlossenen Habilitationsschrift die verfassungsrechtliche Dimension der deutschen Handelsflotte aufgezeigt zu haben.

Zu Beginn seiner Untersuchung widmet sich Dörr der Verfassungsgeschichte des deutschen Handelsflottenrechts. Über die deutsche Hanse, den deutschen Bund, den Norddeutschen Bund, die Reichsverfassung von 1871 und die Weimarer Verfassung gelangt er zur Entstehungsgeschichte des Art. 27 GG. Die redaktionelle Vorbereitung des Art. 27 im parlamentarischen Rat war vor allem von den Schiffbaubeschränkungen durch die Alliierten belastet gewesen, die an einem Wiederaufbau der deutschen Handelsflotte vor allem aus ökonomischen Gründen nicht interessiert waren. So ist der Begriff "deutsche Kauffahrteischiffe" entsprechend dem Zweck von Art. 27, nämlich den Anwendungsbereich völkerrechtlicher Schutzbestimmungen für Handelsschiffe zu umreißen, als alle zum Erwerb durch Seefahrt bestimmten Schiffe erfassend zu verstehen. Zu Dörrs Abgrenzung zwischen See- und Binnenschiffahrt ist anzumerken, daß letztere auch auf Seeverkehrsstraßen fahren und insoweit dann grundsätzlich zur deutschen Handelsflotte gezählt werden müßten, wenn man sie nicht schutzlos stellen wollte.

Im 3. Kapitel erörtert der Verfasser ausführlich die Frage der Flaggenhoheit im Völkerrecht, die die Regeln der Staatszugehörigkeit von Seeschiffen betrifft. So besitzt ein Seeschiff nach geltendem Völkerrecht die Zugehörigkeit zu einem Staat, dessen Flagge es zu führen berechtigt ist. Der Umfang der staatlichen Flaggenhoheit ist jedoch nur auf der Hohen See unbeschränkt. In den Küstengewässem, den Eigengewässem, den Meerengen und den sonstigen internationalen Schiffahrtsstraßen (wie z.B. der Nord-Ostsee-Kanal) ist die Flaggenhoheit zugunsten des Küstenstaates eingeschränkt. Es besteht zwar in der Regel ein Recht der friedlichen Durchfahrt, aber ansonsten sind die Passageregeln des Küstenstaates zu beachten.

Die eigentliche verfassungsrechtliche Diskussion führt der Verfasser in bezug auf die staatliche Pflicht zum Schutz der deutschen Seehandelsschiffe. Ausgehend von der Feststellung, $\mathrm{da}$ Art. 27 als Gewährleistungsnorm die Einrichtung einer einheitlichen deutschen Handelsflotte garantiert, folgert er einen begrenzten Schutz gegen gesetzgeberische Eingriffe (Abwehrfunktion der Einrichtungsgarantie) sowie die Pflicht des Staates, den Bestand und die Funktionsfähigkeit der deutschen Handelsflotte zu schützen. Angesichts des kontinuierlichen Rückgangs der Gesamttonage der deutschen Handelsflotte sieht der Verfasser den Staat hier zu aktiven Schritten verpflichtet. Er stellt daher die Frage, ob die Bundesrepublik das Problem der Ausflaggung, die seiner Ansicht nach dazu geführt habe, $\mathrm{da} \beta$ die deutsche Handelsflotte ihre unverzichtbaren Dienste im Krisen- und Konfliktfall nicht mehr erfüllen könne, mit geeigneten Maßnahmen bekämpft habe. 
Hierzu konnte der Verfasser, der sein Manuskript 1987 abgeschlossen hat, offenbar die unmittelbar danach einsetzende Diskussion um das deutsche Zweitregister nicht mehr würciigen. Mit dem inzwischen verabschiedeten "Gesetz zur Einführung des internationalen Schiffregisters" ist für Schiffe, die im internationalen Seeverkehr eingesetzt werden, die Möglichkeit einer Eintragung in ein beim Bundesverkehrsministerium geführtes zusätzliches Register (das "Erstregister" ist die Eintragung des Schiffes in das Schiffsregister des Amtsgerichts) eröffnet worden. Es besteht seither die Möglichkeit, auf Schiffen unter deutscher Flagge ausländische Seeleute zu den Tarifheuem ihrer Heimatländer zu beschäftigen. Die mit den deutschen Gewerkschaften (ÖTV) ausgehandelten Löhne müssen also nicht bezahlt werden. Die OTV hat daher im März 1990 gegen das Gesetz Verfassungsbeschwerde eingelegt. Neben dem Vorwurf, daß damit gegen den allgemeinen Gleichheitsgrundsatz des Art. 3 GG und die Koalitionsfreiheit und Tarifhoheit gemäß Art. 9 GG verstoßen worden sei, wird von der OTV vorgetragen, daß mit dem Zweitregister die Bundesrepublik nicht ihrer Schutzpflicht gemäß Art. 27 nachgekommen sei.

Eine weitere Rechtsentwicklung ist bei dem vom Verfasser erwähnten Verordnungsentwurf der EG-Kommission über unlautere Preisbildungspraktiken in der Schiffahrt zu verzeichnen. Am 22.12.1986 hat der Rat die Verordnung verabschiedet. Die unrichtigerweise "Frachtratendumping-VO" genannte Regelung bezieht sich auf die Abwehr von Dienstleistungssubventionen, d.h. Reedereien gewährte marktfremde Vorteile durch Drittstaaten. Der Begriff des marktfremden Vorteils ist entgegen dem ursprünglichen Entwurf in der VO nicht erläutert worden, kann aber direkte und indirekte Subventionen einer Fracht umfassen. Das bislang einzige Verfahren, das zur Zeit beim EuGH einer endgültigen Klärung harrt, ist gegenüber einer koreanischen Reederei durchgeführt worden.

Von dem Schicksal wissenschaftlicher Untersuchungen, die einmal abgeschlossen werden müssen, ist auch Dörr nicht verschont geblieben. Auf deutscher wie auf EG-Ebene sind inzwischen Schutzmaßnahmen für die Handelsflotte eingeführt worden, deren Fehlen Dörr in seiner abschließenden Zusammenfassung ausdrücklich bemängelt. Für den anstehenden Verfassungsstreit über das Zweitregister ist die Habilitationsschrift jedoch hervorragend geeignet, dem entscheidenden Senat das notwendige Rüstzeug zu vermitteln. $\mathrm{Ob}$ das verfassungsrechtliche Monopol, das diese Untersuchung hinsichtlich des Verhältnisses zwischen Grundgesetz und Handelsflotte einnimmt, auch den deutschen Reedern zu einer verbesserten Wettbewerbsposition vor dem Bundesverfassungsgericht und auf Hoher See verhelfen wird, bleibt zu wünschen übrig.

Rolf Scheibach 